\title{
Effect of Material on Crashworthiness for Side Doors and B Pillar Subjected to Euro NCAP Side Impact Crash Test.
}

\begin{abstract}
Investigation in effects of material of vehicle body on crashworthiness by simulation methods helps researches to overcome on practical problems of crash test such as die making, long period of test preparation and financial issues. In this paper the goal is to find out the effect of four proposed material on crashworthiness of side doors and B pillar in Euro NCAP side impact test. Materials have been assigned to side doors and B pillar in vehicle model by using LS DYNA pre/post soft ware. Results obtained using LS DYNA post processing by applying Euro NCAP initial conditions. Results show that the best material among mentioned materials for B pillar which can be propose is AISI1006 and best material for side doors is strength steel 204M.
\end{abstract}

Keyword: Crashworthiness; Side impact; Crash simulation; NCAP 